\title{
Long-term clinical outcomes of type 1 vs. type 2 myocardial infarction in patients who underwent angiography: data from the Korea acute myocardial infarction-national institute of health registry
}

\author{
Xiongyi Han ${ }^{1,2} \wedge$, Myung Ho Jeong ${ }^{1 \wedge}$, Liyan Bai ${ }^{1,2} \wedge$, Joon Ho Ahn ${ }^{1 \wedge}$, Dae Young Hyun ${ }^{1 \wedge}$, \\ Kyung Hoon Cho ${ }^{1} \wedge$, Min Chul Kim ${ }^{1 \wedge}$, Doo Sun Sim ${ }^{1} \wedge$, Young Joon Hong ${ }^{1 \wedge}$, Ju Han Kim ${ }^{1 \wedge}$, \\ Youngkeun $\mathrm{Ahn}^{1 \wedge}$; other KAMIR-NIH Registry Investigators \\ ${ }^{1}$ Department of Cardiology, Chonnam National University Hospital, Gwangju, Republic of Korea; ${ }^{2}$ Department of Cardiology, Yanbian University \\ Hospital, Yanji, China \\ Contributions: (I) Conception and design: X Han, MH Jeong; (II) Administrative support: X Han, L Bai, JH Ahn, DY Hyun, KH Cho; (III) Provision \\ of study materials or patients: X Han, L Bai, MH Jeong; (IV) Collection and assembly of data: X Han, L Bai, KH Cho, DS Sim; (V) Data analysis \\ and interpretation: X Han; (VI) Manuscript writing: All authors; (VII) Final approval of manuscript: All authors. \\ Correspondence to: Dr. Myung Ho Jeong, MD, PhD, FACC, FAHA, FESC, FSCAI, FAPSIC. Principal Investigator of Korea Acute Myocardial \\ Infarction Registry, Heart Research Center, Chonnam National University Hospital and Medical School, 671 Jaebongro, Dong-gu, Gwangju 61469, \\ Republic of Korea. Email: myungho@chollian.net.
}

\begin{abstract}
Background: In the cause of increase the clinical research observational evidence on type 2 myocardial infraction (T2MI), the study compared the characteristics of T2MI and T1MI with respect to major adverse cardiac events (MACE) and mortality as long-term outcomes from a large, nationwide, prospective Korean cohort registry.

Methods: From 13,105 consecutively enrolled individuals in the Korea Acute Myocardial InfarctionNational Institute of Health (KAMIR-NIH) registry between October 2011 and December 2015, 11,053 acute myocardial infarction (AMI) patients were divided into the T1MI $(n=10,545)$ and T2MI $(n=508)$ groups. All patients completed $\geq 3$ years of follow-up.

Results: The Kaplan-Meier curve analysis and Cox proportional hazard regression models showed that the cumulative rate of MACE was similar between the 2 groups $(11.4 \%$ vs. $13.4 \%, \log$-rank $\mathrm{P}=0.185)$ at 3 years. However, the T2MI group showed higher rates of all-cause death [12.6\% vs. 9.4\%, log-rank $\mathrm{P}=0.019$; hazard ratio (HR), 1.42; 95\% CI: $1.08-1.85 ; \mathrm{P}=0.012$ ] and non-cardiac death (3.5\% vs. 5.3\%, log-rank $\mathrm{P}=0.043$; HR, 1.55; 95\% CI: 1.01-2.37; P=0.043) than the T1MI group. Male sex (HR 1.540; 95\% CI: 1.218-1.947, $\mathrm{P}<0.001)$, old age ( $\geq 65$ years; HR, 3.546; 95\% CI: 2.645-4.753, $\mathrm{P}<0.001)$, low hemoglobin level $(<12 \mathrm{~g} / \mathrm{dL}$; HR, 2.335; 95\% CI: 1.841-2.961, $\mathrm{P}<0.001)$, high heart rate ( $>100$ beats/min; HR, 1.852; 95\% CI: 1.436-2.388, $\mathrm{P}<0.001)$, low glomerular filtration rate (GFR) $\left(<60 \mathrm{~mL} / \mathrm{min} / 1.73 \mathrm{~m}^{2} ; \mathrm{HR}, 2.373 ; 95 \%\right.$ CI: 1.874-3.005, $\mathrm{P}<0.001$ ), high body mass index ( $>25 \mathrm{~kg} / \mathrm{m}^{2}$; HR, 0.644; 95\% CI: 0.514-0.805, $\left.\mathrm{P}<0.001\right)$, and low left ventricular ejection fraction (LVEF) $(<40 \%$; HR, 1.487; 95\% CI: $1.095-2.020, \mathrm{P}=0.011)$ were the independent predictors for 3-year non-cardiac mortality.
\end{abstract}

\footnotetext{
^ ORCID: Xiongyi Han, 0000-0002-8650-2954; Myung Ho Jeong, 0000-0003-2424-810X; Liyan Bai, 0000-0003-2542-0442; Joon Ho Ahn, 0000-0003-3465-6596; Dae Young Hyun, 0000-0002-0038-0125; Kyung Hoon Cho, 0000-0002-0377-6352; Min Chul Kim, 00000001-6026-1702; Doo Sun Sim, 0000-0003-4162-7902; Young Joon Hong, 0000-0003-0192-8161; Ju Han Kim, 0000-0002-3186-0770; Youngkeun Ahn, 0000-0003-2022-9366.
} 


\begin{abstract}
Conclusions: Although the 2 groups did not differ in MACE, the total mortality rate was higher in T2MI than in T1MI, especially non-cardiac mortality. The independent predictors for non-cardiac mortality were male sex, old age, anemia, low GFR, tachycardia, obesity, and low LVEF.
\end{abstract}

Keywords: Angiography; myocardial infarction; prognosis

Submitted Jul 15, 2021. Accepted for publication Oct 25, 2021.

doi: $10.21037 / \mathrm{cdt}-21-434$

View this article at: https://dx.doi.org/10.21037/cdt-21-434

\section{Introduction}

According to the fourth universal definition in 2018, acute myocardial infarction (AMI) is divided into 5 types (1). Among them, the type 1 and type 2 myocardial infarction (T1MI and T2MI, respectively) are the two more common type MI. T1MI is due to acute atherothrombotic disease involving plaque rupture or erosion in the coronary artery, whereas T2MI is a mismatch between oxygen supply and demand, resulting in a relative myocardial ischemic state, without acute atherothrombotic plaque disruption (1-3). The 2 types of MI with completely different pathophysiologies are difficult to distinguish in the clinical setting because they manifest very similar chest pain symptoms. Even with laboratory tests, it is difficult to make a differential diagnosis before coronary angiography. The pathogenesis of T2MI is particularly complex, and the lack of myocardial blood supply can have many causes. Previous studies reported different morbidity and mortality rates. T2MI was reported to account for $1.6-74 \%$ of all AMI cases $(4,5)$. Moreover, most study results have indicated that the prognosis of T2MI is not as good as that of T1MI (6-9). In contrast, a study in the Japanese population found no difference in long-term mortality between T1MI and T2MI, and T2MI accounted for only $5 \%$ of the study cases (10). The incidence of T2MI is low in epidemiological studies on this condition; however, these studies have limitations and different diagnostic criteria, judgment processes, detection methods with boundary values, and study populations. Therefore, the establishment of uniform and strict diagnostic criteria for T2MI is of great significance to the epidemiological study of this condition. Since the traditional concept of T2MI was first described in 2007 (11), there have been many improvements in the past 10 years. However, compared with the almost perfect treatment of T1MI, the results of various studies on T2MI still have many differences. Moreover, in clinical practice, not much attention is paid to distinguishing the appearance of T2MI, and the understanding of this disorder is not perfect, which directly affect the subsequent treatment plan. Accordingly, the long-term prognosis of T2MI and the factors affecting the prognosis at this stage are still unclear. Therefore, this study was conducted to compare the prognosis of T1MI and T2MI after angiography in a Korean population, and to investigate the main independent predictors affecting the prognosis.

We present the following article in accordance with the STROBE reporting checklist (available at https://cdt. amegroups.com/article/view/10.21037/cdt-21-434/rc).

\section{Methods}

\section{Population and study design}

The study population was selected from the Korea Acute Myocardial Infarction-National Institute of Health (KAMIR-NIH) registry between October 2011 and December 2015. KAMIR-NIH is a nationwide prospective multicenter cohort registry involving 20 tertiary hospitals with resources to perform percutaneous coronary intervention (PCI) in South Korea. The registry was designed to capture the treatment practices for and outcomes of patients with AMI in the real-life setting (12). A total of 13,105 patients were enrolled in the KAMIRNIH registry during the study period. Among them, we excluded patients with no coronary angiography $(n=209)$, procedure-related PCI $(\mathrm{n}=736)$ or coronary artery bypass grafting ( $n=37)$, cardiogenic shock ( $n=727)$, or loss to followup and incomplete data $(n=343)$. Finally, the remaining 11,053 patients with AMI who underwent angiography were divided into the T1MI $(\mathrm{n}=10,545)$ and T2MI $(\mathrm{n}=508)$ groups (Figure 1). All study patients completed the 3-year interviews, medical record reviews, or cell phone followup for outcomes. The study design was approved by the institutional ethics committee.

AMI was diagnosed based on clinical manifestations, 


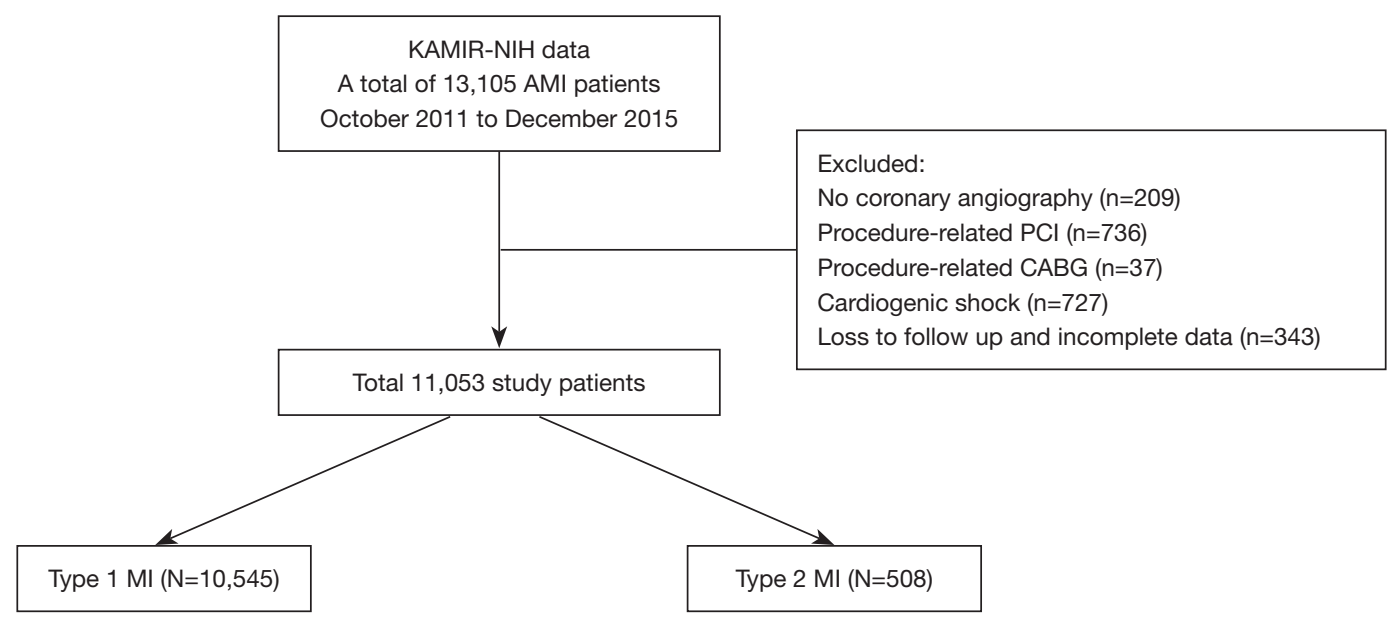

Figure 1 Flowchart of the study patients. KAMIR-NIH, Korean Acute Myocardial Infarction Registry-National Institutes of Health; AMI, acute myocardial infraction; PCI, percutaneous coronary intervention; CABG, coronary artery bypass grafting; MI, myocardial infraction.

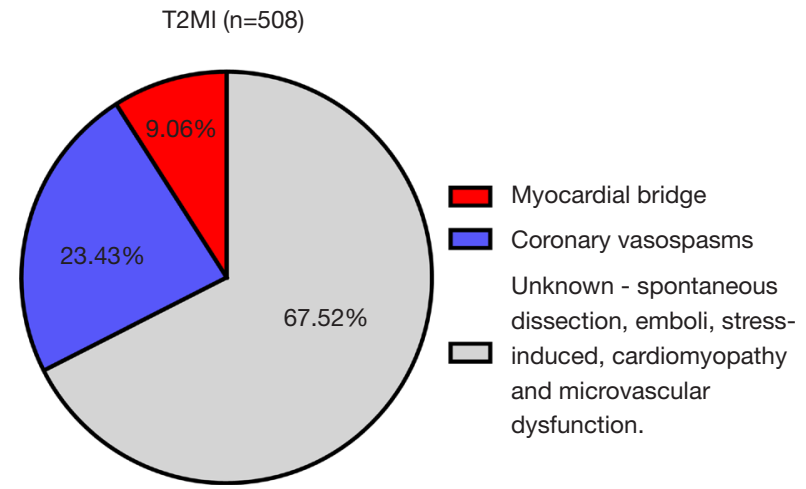

Figure 2 Percentage chart of T2MI patients. T2MI, type 2 myocardial infarction.

elevated levels of myocardial biomarkers [including creatine kinase-MB and troponin (TN)-I or TN-T], and 12 lead electrocardiogram changes (including ST-segment migration and pathological Q-wave development) (12). T1MI group was defined as the detection of an increase and/or a decrease in Tn-I or Tn-T values, (with at least one value being higher than the 99th percentile of the upper reference limit) and at least one of the following findings: AMI symptoms, new ischemic changes on echocardiography, pathological Q-wave development, new loss of viable myocardium or new local wall motion abnormalities consistent with an ischemic etiology on imaging, or obvious coronary artery stenosis or total occlusion detected on coronary angiography (including intracoronary imaging) (13). The T2MI group was defined as patients with increased and/or decreased Tn-I or Tn-T values (with at least one value exceeding the 99th percentile of the upper reference limit), evidence that the imbalance between myocardial oxygen supply and demand was not related to coronary thrombosis, and at least one of the following findings: AMI symptoms, new ischemic changes on echocardiography, pathological Q-wave development, new loss of viable myocardium or new regional wall motion abnormalities consistent with an ischemic etiology on imaging (1), and insignificant stenosis on coronary angiography. On the basis of angiography findings, the causes of AMI in patients with T2MI were coronary vasospasms $(23.43 \%)$ and myocardial bridge (9.06\%). However, $67.52 \%$ of the patients had unknown causes of T2MI, which possibly included idiopathic events (spontaneous dissection), myocardial bridge, emboli, stressinduced, cardiomyopathy and microvascular dysfunction (Figure 2).

The primary outcome of this study was the occurrence of clinical events within 3 years, defined as all-cause death, cardiac death, non-cardiac death, recurrent MI, repeat PCI, and major adverse cardiac events (MACE; defined as the composite of all-cause death, recurrent MI, and repeat PCI). Additionally, we investigated the independent predictors that affect these clinical events.

\section{Statistical analysis}

Continuous variables are expressed as mean and standard deviation values or medians with the interquartile range 
and were compared using the unpaired $t$-test or MannWhitney U-test, as appropriate. All categorical variables are expressed as numbers and percentages and analyzed using the chi-square test or Fisher's exact test. All available baseline clinical, laboratory, and pharmaceutical variables were tested. Survival analysis was performed using the Kaplan-Meier method and Cox proportional hazard regression model to compare the MACE risk. On the basis of the Cox proportional hazard regression models, significant variables with $\mathrm{P}<0.10$ on univariate analysis were entered into the multivariate Cox regression model. Multivariate analysis was performed using the backward regression method and included old age ( $\geq 65$ years), high heart rate $(>100$ beats/min), male sex, high body mass index $\left(>25 \mathrm{~kg} / \mathrm{m}^{2}\right)$, hypertension, diabetes mellitus, dyslipidemia, current smoking, low estimated glomerular filtration rate (eGFR; $<60 \mathrm{~mL} / \mathrm{min} / 1.73 \mathrm{~m}^{2}$ ), low hemoglobin level $(<12 \mathrm{~g} / \mathrm{dL}$ ), and low left ventricular ejection fraction (LVEF; $<40 \%)$. Multivariate Cox regression analysis was performed to obtain hazard ratios (HRs) and $95 \%$ confidence intervals (CIs) in the adjusted survival analysis.

All analyses were 2-tailed, with $\mathrm{P}<0.05$ considered significant, and statistical analyses were performed using IBM-SPSS Statistics for Windows software (version 25.0; SPSS Inc., Chicago, IL, USA).

\section{Ethical statement}

The study was conducted in accordance with the Declaration of Helsinki (as revised in 2013). The institutional review board of all individual participating centers approved the study protocol, and the approval number of Chonnam National University Hospital was CNUH-2011-172. All patients enrolled completed the informed consent form.

\section{Results}

The T1MI and T2MI groups included 10,545 (95.4\%) and $508(4.6 \%)$ patients, respectively. The mean patient age was similar between the T1MI and T2MI groups $(63.56 \pm 12.55$ vs. $62.49 \pm 12.69$ years, $\mathrm{P}=0.060)$, and $48.3 \%$ and $46.9 \%$ of the patients were aged $\geq 65$ years in the T1MI and T2MI group, respectively. The T1MI group were male more often, were more likely to have a smoking habit and be presented with typical chest pain compared with the T2MI group. Moreover, the diagnosis rate of ST-segment elevation MI was higher in the T1MI group than in the T2MI group (48.6\% vs. $13.8 \%, \mathrm{P}<0.001)$.
However, The T1MI group were more likely to have a history of MI, angina, and heart failure compared with the T2MI group. Further, the mean LVEF was lower in the T1MI group than in the T2MI group $(52.37 \% \pm 10.74 \%$ vs. $58.28 \% \pm 11.16 \%, \mathrm{P}<0.001)$. There were more patients in the T1MI group than in the T2MI group with Killip class III disease. However, fewer patients in the T1MI group than in the T2MI group had a low Killip class (I and II). According to laboratory findings, the mean white blood cell count, neutrophil cell count, platelet count, and hemoglobin level; total cholesterol, low-density lipoprotein cholesterol, and glucose levels and creatine kinase-MB, Tn$\mathrm{I}$, and hemoglobin A1c were higher in the T1MI group than in the T2MI group. However, the mean lymphocyte cell count, high-density lipoprotein cholesterol level, GFR, and P2Y12 reaction units were lower in the T1MI group than in the T2MI group (Table 1).

In terms of coronary angiography, the frequency of performing the procedure for $\mathrm{PCI} /$ plain old balloon angioplasty was higher in the T1MI group than in the T2MI group (95.6\% vs. $6.5 \%, \mathrm{P}<0.001)$. The proportions of patients with multivessel disease and lesions in each of the target vessels (left main/left anterior descending/left circumflex/right coronary artery) were also higher in the T1MI group than in the T2MI group. A higher number of patients with American College of Cardiology/American Heart Association type 2B or C lesions and poor initial thrombolysis in MI flow (0/1) were found in the T1MI group than in the T2MI group $(82.1 \%$ vs. $4.9 \%, \mathrm{P}<0.001$ and $54.1 \%$ vs. $3.9 \%$, respectively; $\mathrm{P}<0.001)$. Further, implanted stents were more prevalent in the T1MI group than in the T2MI group $(89.6 \%$ vs. $6.3 \%, \mathrm{P}<0.001)$, and about $86.8 \%$ of patients underwent placement of drugeluting stents in the T1MI group. With respect to medical therapy, aspirin $(99.8 \%$ vs. $96.5 \%)$, statins $(93.2 \%$ vs. $78.0 \%$ ), beta-blockers ( $84.9 \%$ vs. $37.6 \%$ ), and angiotensinconverting enzyme inhibitors/angiotensin II receptor blockers $(79.9 \%$ vs. $50.2 \%)$ were used more in the T1MI group than in the T2MI group; however, calcium channel blockers $(6.3 \%$ vs. $47.8 \%)$ were used less often in the T1MI group than in the T2MI group (Table 2).

Kaplan-Meier curve analysis and Cox proportional hazard regression models showed that the cumulative rate of all-cause death was lower in the T1MI group ( $\mathrm{n}=996)$ than in the T2MI group ( $\mathrm{n}=64)(9.4 \%$ vs. $12.6 \%, \log -$ rank $\mathrm{P}=0.019$; HR, 1.42; 95\% CI: $1.08-1.85 ; \mathrm{P}=0.012$ ). Moreover, non-cardiac mortality was also lower in the T1MI group $(n=365)$ than in the T2MI group $(n=27)(3.5 \%$ 
Table 1 Clinical baseline and laboratory characteristics of T1MI and T2MI patients

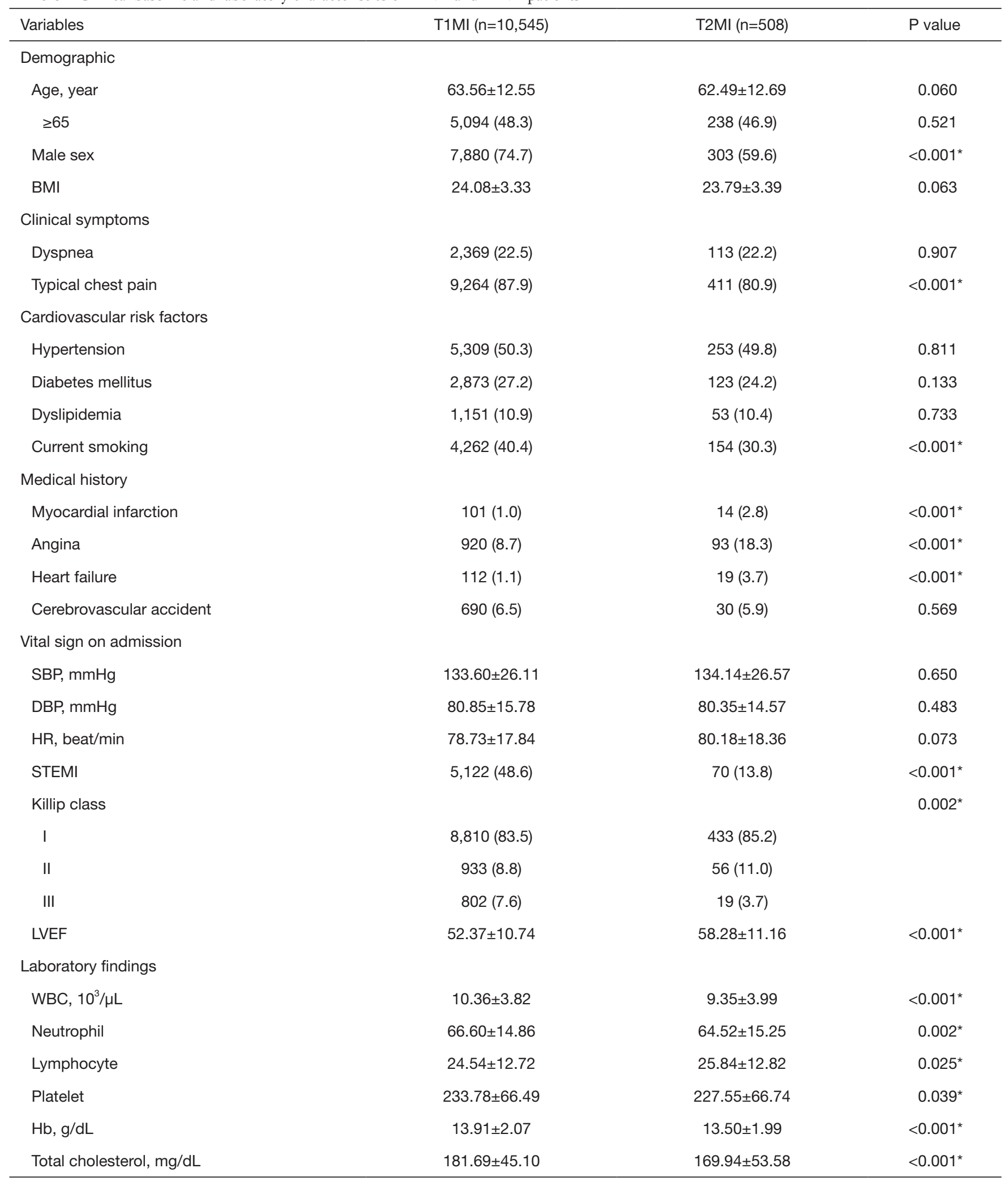

Table 1 (continued) 
Table 1 (continued)

\begin{tabular}{|c|c|c|c|}
\hline Variables & T1MI $(n=10,545)$ & T2MI $(n=508)$ & $P$ value \\
\hline HDL-cholesterol, mg/dL & $42.91 \pm 11.68$ & $47.23 \pm 14.02$ & $<0.001^{*}$ \\
\hline LDL-cholesterol, mg/dL & $114.81 \pm 39.28$ & $102.05 \pm 37.24$ & $<0.001^{*}$ \\
\hline Glucose & $164.55 \pm 74.64$ & $149.58 \pm 70.85$ & $<0.001^{*}$ \\
\hline GFR, $\mathrm{mL} / \mathrm{min} / 1.73 \mathrm{~m}^{2}$ & $89.66 \pm 40.05$ & $98.01 \pm 47.20$ & $<0.001^{*}$ \\
\hline Hs-CRP, mg/dL & $1.44 \pm 5.95$ & $1.65 \pm 5.32$ & 0.596 \\
\hline Peak CK-MB, ng/mL & $110.23 \pm 144.42$ & $36.50 \pm 65.60$ & $<0.001^{*}$ \\
\hline Peak Troponin-I, ng/mL & $45.50 \pm 103.81$ & $10.18 \pm 24.77$ & $<0.001^{*}$ \\
\hline PRU & $195.70 \pm 109.02$ & $238.81 \pm 107.72$ & $0.017^{*}$ \\
\hline ARU & $457.98 \pm 73.51$ & $469.49 \pm 72.28$ & 0.358 \\
\hline
\end{tabular}

Data are expressed as $\mathrm{n}(\%)$ or mean $\pm \mathrm{SD}$. *, P value $<0.05$ denotes statistical significance. T1Ml, type 1 myocardial infarction; T2MI, type 2 myocardial infarction; BMI, body mass index; SBP, systolic blood pressure; DBP, diastolic blood pressure; HR, heart rate; STEMI, STsegment elevation myocardial infarction; LVEF, left ventricular ejection fraction; WBC, white blood cell; Hb, hemoglobin; HDL, high-density lipoprotein; LDL, low-density lipoprotein; GFR, glomerular filtration rate; Hs-CRP, high-sensitivity C-reactive protein; CK, creatine kinase; BNP, brain natriuretic peptide; NT-pro-BNP, N-terminal pro-brain natriuretic peptide; PRU, P2Y12 reaction units; ARU, aspirin reaction units.

vs. $5.3 \%$, log-rank $\mathrm{P}=0.024$; HR, 1.55; 95\% CI: $1.01-2.37$; $\mathrm{P}=0.043$; Figure 3 and Table 3). The non-cardiac mortality rate was statistically significantly different between the 2 groups and was a key factor affecting the rate of all-cause mortality. Therefore, we also assessed the independent predictors for non-cardiac mortality. Table 4 summarizes the results of Cox regression multivariate analysis of the independent predictors for 3-year non-cardiac mortality. The results showed that male sex (HR, 1.540; 95\% CI: $1.218-1.947 ; \mathrm{P}<0.001$ ), old age ( $\geq 65$, years; HR, $3.546 ; 95 \%$ CI: 2.645-4.753; P<0.001), low hemoglobin level $(<12 \mathrm{~g} / \mathrm{dL}$; HR, 2.335; 95\% CI: 1.841-2.961; $\mathrm{P}<0.001)$, high heart rate ( $>100$ beats/min; HR, 1.852; 95\% CI: $1.436-2.388$; $\mathrm{P}<0.001)$, low GFR $\left(<60 \mathrm{~mL} / \mathrm{min} / 1.73 \mathrm{~m}^{2} ; \mathrm{HR}, 2.373\right.$; 95\% CI: $1.874-3.005 ; \mathrm{P}<0.001)$, high body mass index (>25 kg/m²; HR, 0.644; 95\% CI: 0.514-0.805; P<0.001), and low LVEF (<40\%; HR, 1.487; 95\% CI: 1.095-2.020; $\mathrm{P}=0.011)$ were the independent predictors for 3 -year non-cardiac mortality. The causes of in-hospital deaths in patients with T2MI are shown in Figure 4. Cardiac death and non-cardiac death occurred in 6 and 5 patients, respectively, with pump failure being the most frequent cause of death, followed by sepsis and multiorgan failure. In-hospital all-cause death accounted for only $2.2 \%(n=11)$, and non-cardiac death accounted for only $1.0 \%(\mathrm{n}=5)$.

\section{Discussion}

The main findings of our study were that the prevalence of $\mathrm{T} 2 \mathrm{MI}$ was $4.6 \%$, and $\mathrm{T} 2 \mathrm{MI}$ was associated with poorer outcomes than T1MI. A significant difference in mortality was found, and the most important factor leading to the difference between the 2 groups was non-cardiac death during the long-term clinical outcomes of 3 years. Further, in-hospital mortality accounted for only $2.2 \%$ and noncardiac mortality accounted for only $1.0 \%$ in the T2MI group. The independent predictors for non-cardiac mortality at 3 years in the T1MI and T2MI groups were male sex, old age, anemia, low GFR, tachycardia, obesity, and low LVEF.

Many previous studies have reported the prognosis of T2MI, and most of them agree that the prognosis of T2MI is poorer than that of T1MI $(7,9,14-16)$. Our results are also consistent with this concept. Additionally, in a recent 
Table 2 Coronary angiography and medication characteristics in T1MI and T2MI patients

\begin{tabular}{|c|c|c|c|}
\hline Variables & $\mathrm{T} 1 \mathrm{MI}(\mathrm{n}=10,545)$ & T2MI (n=508) & $P$ value \\
\hline Procedure for $\mathrm{PCl} / \mathrm{POBA}$ & $10,083(95.6)$ & $33(6.5)$ & $<0.001^{*}$ \\
\hline Multivessel disease & $1,947(18.5)$ & $5(1.0)$ & $<0.001^{*}$ \\
\hline Target vessel in coronary artery & & & $<0.001^{*}$ \\
\hline Left anterior descending & $4,836(45.9)$ & $13(2.6)$ & \\
\hline Left circumflex & $1,789(17.0)$ & $7(1.4)$ & \\
\hline Right coronary artery & $3,262(30.9)$ & $12(2.4)$ & \\
\hline ACC/AHA B2/C lesion & $8,661(82.1)$ & $25(4.9)$ & $<0.001^{*}$ \\
\hline \multicolumn{4}{|l|}{ TIMI flow grade } \\
\hline Initial TIMI flow 0/1 & $5,701(54.1)$ & $20(3.9)$ & $<0.001^{*}$ \\
\hline Final TIMI flow 3 & $9,754(92.5)$ & $33(6.5)$ & $<0.001^{\star}$ \\
\hline \multicolumn{4}{|l|}{ Medical treatment, n (\%) } \\
\hline Aspirin & $10,525(99.8)$ & $490(96.5)$ & $<0.001^{*}$ \\
\hline Clopidogrel & $8,162(77.4)$ & $412(81.1)$ & 0.051 \\
\hline Statin & $9,827(93.2)$ & $396(78.0)$ & $<0.001^{*}$ \\
\hline Beta-blocker & $8,951(84.9)$ & $191(37.6)$ & $<0.001^{*}$ \\
\hline
\end{tabular}

*, $\mathrm{P}$ value $<0.05$ denotes statistical significance. T1MI, type 1 myocardial infarction; T2MI, type 2 myocardial infarction; PCl, percutaneous coronary intervention; POBA, plain old balloon angioplasty; ACC, American College of Cardiology; AHA, American Heart Association; DES, dual eluting stent; $A C E I$, angiotensin-converting enzyme inhibitor; ARB, angiotensin II receptor blocker.

study, the higher long-term mortality of T2MI than T1MI was caused by early mortality (3). In terms of in-hospital mortality or short-term mortality, Putot et al. reported that the mortality rate of T2MI (14\%) was higher than that of T1MI $(6.1 \%)$ in patients who died of non-cardiac causes (17). Moreover, Smilowitz et al. found that the inhospital mortality rate in patients with T2MI who dies of non-cardiac causes was $>80 \%$ (18). A recent study reported that T2MI had a lower risk of in-hospital mortality (odds ratio: 0.57 ) and 30-day readmission for MI (odds ratio: 0.46) (19). Although their results are somewhat different from ours in terms of the rates of in-hospital death $(2.2 \%)$ and recurrent MI (only 4 patients at 3 years, $0.8 \%$ ) in patients with T2MI, these outcomes might have differed due to the different treatments at each hospital and the severity of each patient's condition. However, the main reason for the higher death rate in T2MI than in T1MI was clearly non-cardiovascular factors. Whereas the use of standard prognostic therapies was lower in T2MI, the difficulty lies in determining the primary disease, especially in high-risk patients with multiple diseases. In our study, aspirin and $\mathrm{P} 2 \mathrm{Y} 12$ inhibitors were used at a high rate (Table 2). The effect of antithrombotic and/or antiplatelet therapy and the effect of reperfusion on patients with T2MI may be beneficial in those with mild atherosclerosis and coronary artery stenosis; however, the benefits for patients without plaque rupture are uncertain $(14,20)$. Therefore, anticoagulant drugs should be used with more caution. 

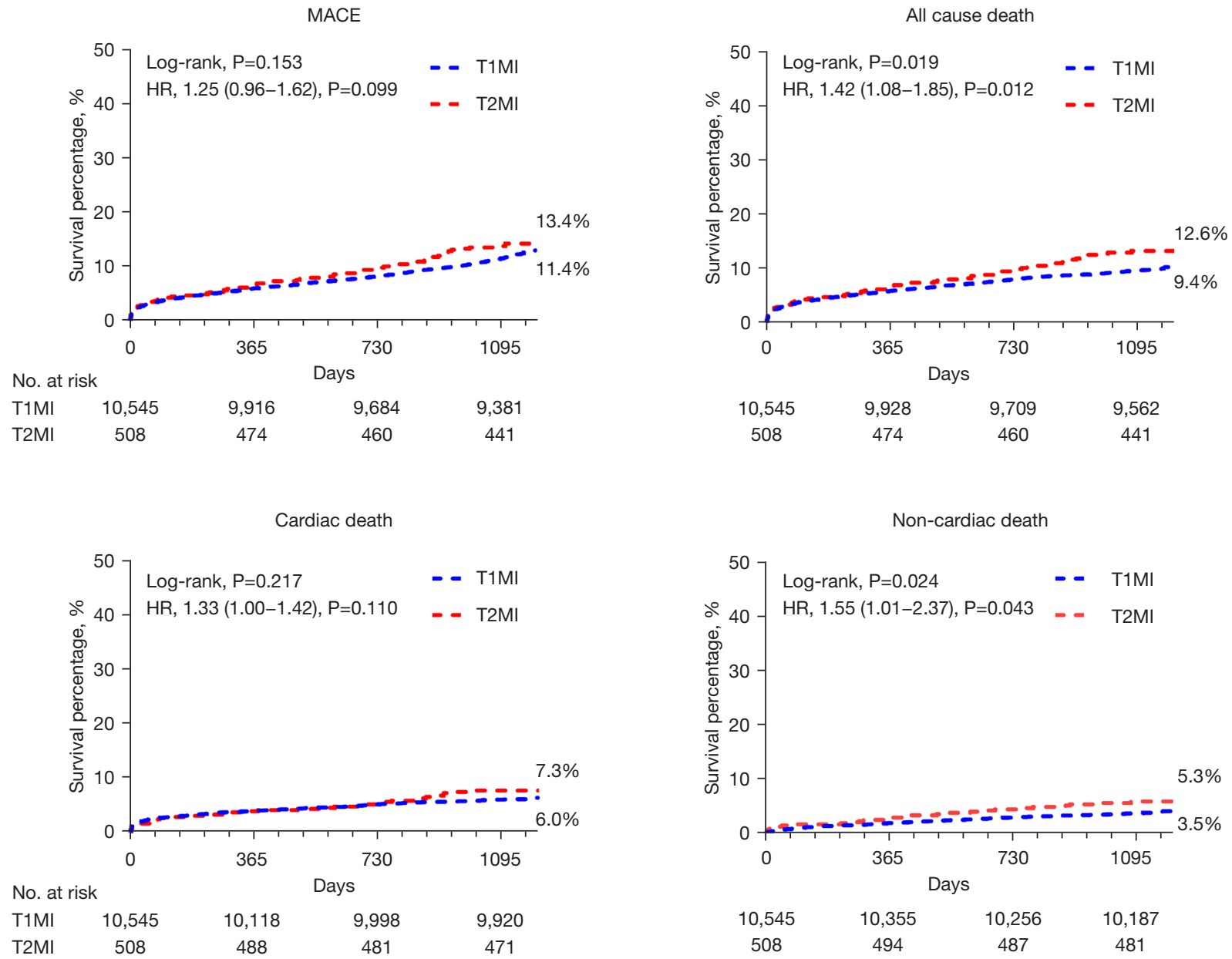

Figure 3 The major adverse cardiac events, all-cause death, cardiac death, and non-cardiac death in the Kaplan-Meier curves and the HR values. HR, hazard ratio; MACE, major adverse cardiac events; T1MI, type 1 myocardial infarction; T2MI, type 2 myocardial infarction.

Table 3 Clinical outcomes (unadjusted and adjusted) at 3 years among T1MI and T2MI patients

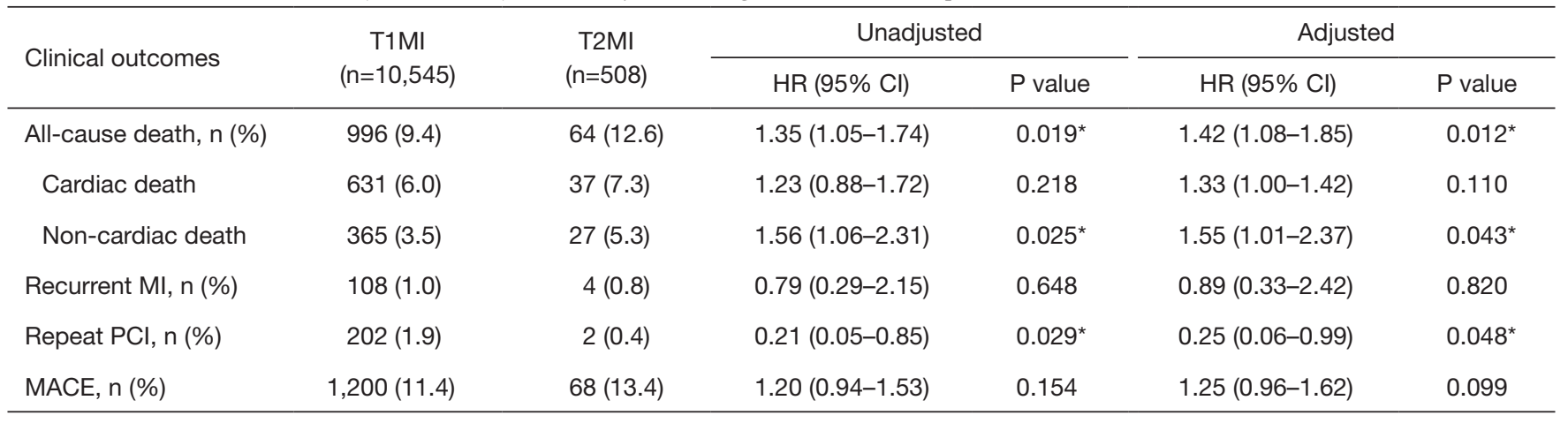

*, $\mathrm{P}$ value $<0.05$ denotes statistical significance. Adjusted for age, sex, diabetes, hypertension, dyslipidemia, GFR, smoking, BMI, LVEF, heart rate and anemia. MI, myocardial infarction; T1MI, type 1 myocardial infraction; T2MI, type 2 myocardial infraction; PCI, percutaneous coronary intervention; MACE, major adverse cardiac events. 
Table 4 Univariate and multivariate analysis for predictors of 3-year non-cardiac death

\begin{tabular}{|c|c|c|c|c|}
\hline Risk factor in non-cardiac death & \multicolumn{2}{|c|}{ Univariate analysis } & \multicolumn{2}{|c|}{ Multivariate analysis } \\
\hline Male & $0.630(0.512-0.776)$ & $<0.001^{*}$ & $1.540(1.218-1.947)$ & $<0.001^{\star}$ \\
\hline Age $\geq 65$ years & $5.821(4.468-7.584)$ & $<0.001^{*}$ & $3.546(2.645-4.753)$ & $<0.001^{\star}$ \\
\hline $\mathrm{Hb}<12 \mathrm{~g} / \mathrm{dL}$ & 4.861 (3.983-5.932) & $<0.001^{*}$ & $2.335(1.841-2.961)$ & $<0.001^{\star}$ \\
\hline $\mathrm{BMI} \geq 25 \mathrm{~kg} / \mathrm{m}^{2}$ & $0.453(0.365-0.564)$ & $<0.001^{*}$ & $0.644(0.514-0.805)$ & $<0.001^{\star}$ \\
\hline Hypertension & $1.700(1.385-2.085)$ & $<0.001^{*}$ & $0.946(0.757-1.183)$ & 0.627 \\
\hline Diabetes mellitus & $1.873(1.530-2.293)$ & $<0.001^{*}$ & $1.103(0.883-1.377)$ & 0.388 \\
\hline Dyslipidemia & $0.733(0.513-1.048)$ & 0.088 & & \\
\hline LVEF $<40 \%$ & $2.971(2.338-3.775)$ & $<0.001^{*}$ & 1.487 (1.095-2.020) & $0.011^{*}$ \\
\hline
\end{tabular}

*, P value $<0.05$ denotes statistical significance. $\mathrm{Cl}$, confidence interval; HR, Hazard ratios; Hb, hemoglobin; BMI, body mass index; GFR, glomerular filtration rate; LVEF; left ventricular ejection fraction.

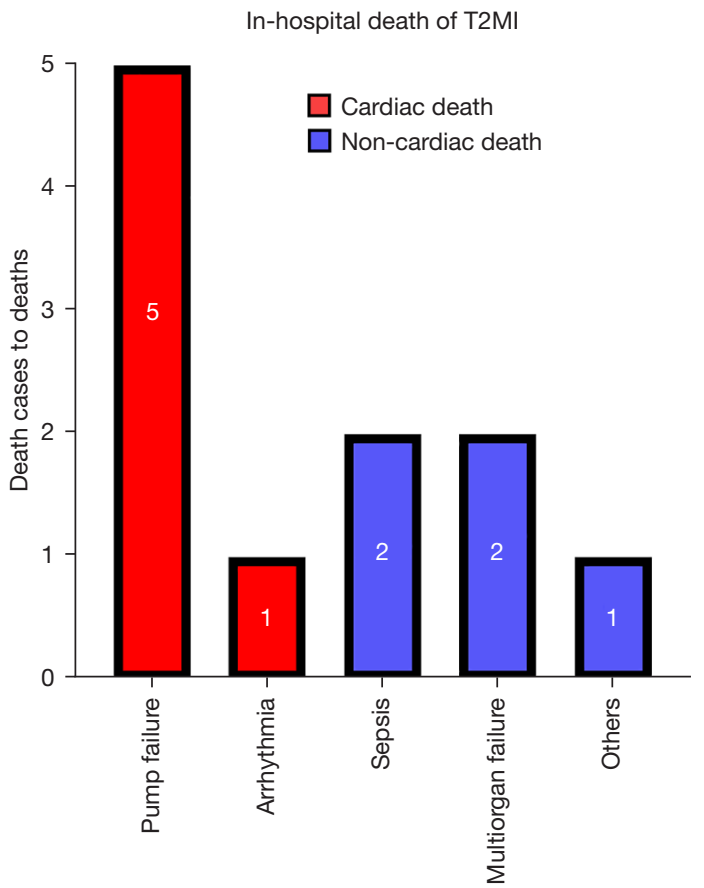

Figure 4 Detailed cause of in-hospital deaths in patients with T2MI. T2MI, type 2 myocardial infarction.

Further, we found that less than half of the patients in the T2MI group used grade 2 preventive medications, except for statin. On the contrary, the usage rate of calcium channel blockers was significantly higher than that of T1MI patients, which may be due to the influence of spasms. Otherwise, T2MI patients often receive specialized treatments for comorbid diseases, such as the use of anticoagulants to manage atrial fibrillation or of diuretics to manage heart failure (21). Therefore, the treatment of $\mathrm{T} 2 \mathrm{MI}$ is very important, and treatment of the primary disease is the key point.

With respect to PCI, it is not recommended for T2MI patients. In our data, only $6.5 \%$ of patients underwent PCI or plain old balloon angioplasty. Functional evaluation of myocardial perfusion for the diagnosis of underlying coronary artery disease following T2MI detection may also be considered (15). In the current clinical practice, patients with T2MI are less likely to undergo coronary angiography (22). Moreover, a retrospective review reported that non-cardiac surgery was the setting most associated with T2MI (23). The possible causes of T2MI were ischemic changes with AMI (ST-segment elevation MI or non-ST-segment elevation MI), without any plaque destruction; at least 1 chronic total occlusion in ischemic patients; or a true non-ischemic cause of reduced ejection fraction, such as an idiopathic, toxic, inflammatory, or arrhythmic cause; and associated silent obstructive coronary artery disease (24). Therefore, we still recommend performing coronary angiography to correctly assess the cause of T2MI, as this is a very useful treatment and 
evaluation method for T2MI.

In our study, a significantly lower frequency of T2MI than T1MI was observed. The proportion of patients with T2MI was only $4.6 \%$, although it may, in fact, be higher. This is because KAMIR is a large database that collects data of patients with AMI and accepts the coronary angiography procedure. Many T2MI patients with no symptoms or with typical spasm may have been passively excluded. Moreover, patients with Takotsubo cardiomyopathy or pulmonary embolism are also excluded. Other studies revealed that the number of men with T2MI was lower than that of women $(3,25,26)$. However, we found that more men than women had T1MI or T2MI, which is very similar to the results of a Japanese study (10). Men in East Asia have a higher rate of MI than men in Western countries. Interestingly, among the independent predictors for cardiovascular disease, hypertension, diabetes mellitus, and hyperlipidemia showed no difference between T2MI and T1MI, and the rate of smoking was significantly higher in T1MI than in T2MI. Additionally, the proportions of patients with a history of MI, angina, and heart failure were higher in T2MI. Therefore, T2MI is a poorer condition than T1MI. Male sex, old age, anemia, low GFR, tachycardia, obesity, and low LVEF were the factors that affected long-term noncardiac mortality in T2MI patients in this study. Previous studies have not identified clear independent predictors (27). However, other studies have shown that old age, renal insufficiency, hemorrhage, severe aortic stenosis, infection, tachycardia, and heart failure can all be predisposing factors of T2MI $(7,22,23)$.

This study had several limitations. First, only patients with AMI in major PCI centers in Korea were considered and the study was not a randomized controlled clinical trial. Moreover, this study had a retrospective design. Second, because KAMIR is a large database that collects the data of patients with AMI and accepts the coronary angiography procedure, patients who have not undergone coronary angiography may not be included in the database. Third, in the long-term follow-up, we only determined whether the deaths were caused by cardiac or non-cardiac diseases. Therefore, it was impossible to elucidate the detailed causes of death. Fourth, we did not assess whether the medical treatment after hospital discharge was reasonable and there was a lack of detailed indicators for the inability to continue oral medications because of various other diseases. Finally, we need more recent, longer, or real-time data to increase the strength of our findings.

\section{Conclusions}

In summary, although there was no difference between the 2 groups in MACE, the T2MI group had a higher mortality than the T1MI group during the 3-year clinical followup. The most important factor leading to the difference between the 2 groups was non-cardiac death. The independent predictors for non-cardiac mortality were male sex, old age, anemia, low GFR, tachycardia, obesity, and low LVEF.

\section{Acknowledgments}

The authors thank the Korea Acute Myocardial Infarction Registry-National Institutes of Health (KAMIR-NIH) investigators: Sung Chul Chae, MD; Jong Hyun Kim, MD; Seung-Ho Hur, MD; Young Jo Kim, MD; In Whan Seong, MD; Donghoon Choi, MD; Jei Keon Chae, MD; Taek Jong Hong, MD; Jae Young Rhew, MD; Doo-Il Kim, MD; In-Ho Chae, MD; Jung Han Yoon, MD; Bon-Kwon Koo, MD; Byung-Ok Kim, MD; Myoung Yong Lee, MD; Kee-Sik Kim, MD; Jin-Yong Hwang, MD; Myeong Chan Cho, MD; Seok Kyu Oh, MD; Nae-Hee Lee, MD; Kyoung Tae Jeong, MD; Seung-Jea Tahk, MD; Jang-Ho Bae, MD; Seung-Woon Rha, MD; Keum-Soo Park, MD; Chong Jin Kim, MD; Kyoo-Rok Han, MD; Tae Hoon Ahn, MD; Moo-Hyun Kim, MD; Ki Bae Seung, MD; Wook Sung Chung, MD; Ju-Young Yang, MD; Chong Yun Rhim, MD; Hyeon-Cheol Gwon, MD; Seong-Wook Park, MD; YoungYoup Koh, MD; Seung Jae Joo, MD; Soo-Joong Kim, MD; Dong Kyu Jin, MD; Jin Man Cho, MD; Sang-Wook Kim, MD; Jeong Kyung Kim, MD; Tae Ik Kim, MD; Deug Young Nah, MD; Si Hoon Park, MD; Sang Hyun Lee, MD; Seung Uk Lee, MD; Hang-Jae Chung, MD; JangHyun Cho, MD; Seung Won Jin, MD; Myeong-Ki Hong, MD; Yangsoo Jang, MD; Jeong Gwan Cho, MD; Hyo-Soo Kim, MD; and Seung Jung Park, MD.

Funding: The research was supported by a research fund (2016-ER6304-02) from the Korea Centers for Disease Control and Prevention.

\section{Footnote}

Reporting Checklist: The authors have completed the STROBE reporting checklist. Available at https://cdt. amegroups.com/article/view/10.21037/cdt-21-434/rc

Data Sharing Statement: Available at https://cdt.amegroups. 
com/article/view/10.21037/cdt-21-434/dss

Peer Review File: Available at https://cdt.amegroups.com/ article/view/10.21037/cdt-21-434/prf

Conflicts of Interest: All authors have completed the ICMJE uniform disclosure form (available at https://cdt.amegroups. com/article/view/10.21037/cdt-21-434/coif). The authors have no conflicts of interest to declare.

Ethical Statement: The authors are accountable for all aspects of the work in ensuring that questions related to the accuracy or integrity of any part of the work are appropriately investigated and resolved. The study was conducted in accordance with the Declaration of Helsinki (as revised in 2013). The institutional review board of all individual participating centers approved the study protocol, and the approval number of Chonnam National University Hospital was CNUH-2011-172. All patients enrolled completed the informed consent form.

Open Access Statement: This is an Open Access article distributed in accordance with the Creative Commons Attribution-NonCommercial-NoDerivs 4.0 International License (CC BY-NC-ND 4.0), which permits the noncommercial replication and distribution of the article with the strict proviso that no changes or edits are made and the original work is properly cited (including links to both the formal publication through the relevant DOI and the license). See: https://creativecommons.org/licenses/by-nc-nd/4.0/.

\section{References}

1. Thygesen K, Alpert JS, Jaffe AS, et al. Fourth universal definition of myocardial infarction (2018). J Am Coll Cardiol 2018;72:2231-64.

2. McCarthy CP, Vaduganathan M, Januzzi JL Jr. Type 2 Myocardial Infarction-Diagnosis, Prognosis, and Treatment. JAMA 2018;320:433-4.

3. Raphael CE, Roger VL, Sandoval Y, et al. Incidence, trends, and outcomes of type 2 myocardial infarction in a community cohort. Circulation 2020;141:454-63.

4. Sandoval Y, Jaffe AS. Type 2 myocardial infarction: JACC review topic of the week. J Am Coll Cardiol 2019;73:1846-60.

5. Melberg T, Burman R, Dickstein K. The impact of the 2007 ESC-ACC-AHA-WHF Universal definition on the incidence and classification of acute myocardial infarction: a retrospective cohort study. Int J Cardiol 2010;139:228-33.

6. Gaggin HK, Liu Y, Lyass A, et al. Incident Type 2 Myocardial Infarction in a Cohort of Patients Undergoing Coronary or Peripheral Arterial Angiography. Circulation 2017;135:116-27.

7. Putot A, Jeanmichel M, Chague F, et al. Type 2 Myocardial Infarction: A Geriatric Population-based Model of Pathogenesis. Aging Dis 2020;11:108-17.

8. Sandoval Y, Thygesen K. Myocardial infarction type 2 and myocardial injury. Clin Chem 2017;63:101-7.

9. Baron T, Hambraeus K, Sundström J, et al. Type 2 myocardial infarction in clinical practice. Heart 2015;101:101-6.

10. Sato R, Sakamoto K, Kaikita K, et al. Long-Term Prognosis of Patients with Myocardial Infarction Type 1 and Type 2 with and without Involvement of Coronary Vasospasm. J Clin Med 2020;9:1686.

11. Thygesen K, Alpert JS, White HD, et al. Universal definition of myocardial infarction. J Am Coll Cardiol 2007;50:2173-95.

12. Kim Y, Jeong MH, Ahn Y, et al. Results of a 10-Year Experience in Korea Using Drug-Eluting Stents During Percutaneous Coronary Intervention for Acute Myocardial Infarction (from the Korea Acute Myocardial Infarction Registry). Am J Cardiol 2018;122:365-73.

13. Thygesen K, Alpert JS, Jaffe AS, et al. Third universal definition of myocardial infarction. J Am Coll Cardiol 2012;60:1581-98.

14. Chapman AR, Shah AS, Lee KK, et al. Long-term outcomes in patients with type 2 myocardial infarction and myocardial injury. Circulation 2018;137:1236-45.

15. Curcio F, Gerundo G, Sasso G, et al. Type 2 myocardial infarction: is it a geriatric syndrome? Aging Clin Exp Res 2020;32:759-68.

16. Sandoval Y, Smith SW, Sexter A, et al. Type 1 and 2 myocardial infarction and myocardial injury: clinical transition to high-sensitivity cardiac troponin I. Am J Med 2017;130:1431-9.e1434.

17. Putot A, Derrida SB, Zeller M, et al. Short-term prognosis of myocardial injury, type 1 , and type 2 myocardial infarction in the Emergency Unit. Am J Med 2017;130:1431-1439.e4.

18. Smilowitz NR, Subramanyam P, Gianos E, et al. Treatment and Outcomes of Type 2 Myocardial Infarction and Myocardial Injury Compared to Type 1 Myocardial Infarction. Coron Artery Dis 2018;29:46-52.

19. McCarthy CP, Kolte D, Kennedy KF, et al. Patient Characteristics and Clinical Outcomes of Type 1 Versus 
Type 2 Myocardial Infarction. J Am Coll Cardiol 2021;77:848-57.

20. López-Cuenca A, Gómez-Molina M, Flores-Blanco PJ, et al. Comparison between type- 2 and type- 1 myocardial infarction: clinical features, treatment strategies and outcomes. J Geriatr Cardiol 2016;13:15-22.

21. Wang G, Zhao N, Zhong S, et al. A systematic review on the triggers and clinical features of type 2 myocardial infarction. Clin Cardiol 2019;42:1019-27.

22. Stein GY, Herscovici G, Korenfeld R, et al. Type-II myocardial infarction-patient characteristics, management and outcomes. PloS One 2014;9:e84285.

23. Smilowitz NR, Weiss MC, Mauricio R, et al. Provoking conditions, management and outcomes of type 2 myocardial infarction and myocardial necrosis. Int $\mathrm{J}$ Cardiol 2016;218:196-201.

Cite this article as: Han $\mathrm{X}$, Jeong $\mathrm{MH}$, Bai L, Ahn JH, Hyun DY, Cho KH, Kim MC, Sim DS, Hong YJ, Kim JH, Ahn Y; other KAMIR-NIH Registry Investigators. Long-term clinical outcomes of type $1 v$ s. type 2 myocardial infarction in patients who underwent angiography: data from the Korea acute myocardial infarction-national institute of health registry. Cardiovasc Diagn Ther 2022;12(1):55-66. doi: 10.21037/cdt$21-434$
24. David C, Bisson A, André C, et al. Prognosis of Type 2 Myocardial Infarction Patients Implanted With a Prophylactic Defibrillator (from the Very-High-Rate Registry). Am J Cardiol 2020;125:1001-5.

25. Arora S, Strassle PD, Qamar A, et al. Impact of Type 2 Myocardial Infarction (MI) on Hospital-Level MI Outcomes: Implications for Quality and Public Reporting. J Am Heart Assoc 2018;7:e008661.

26. Sandoval Y, Smith SW, Schulz KM, et al. Diagnosis of type 1 and type 2 myocardial infarction using a high-sensitivity cardiac troponin I assay with sex-specific 99th percentiles based on the third universal definition of myocardial infarction classification system. Clin Chem 2015;61:657-63.

27. Singh A, Gupta A, DeFilippis EM, et al. Cardiovascular Mortality After Type 1 and Type 2 Myocardial Infarction in Young Adults. J Am Coll Cardiol. 2020;75:1003-13. 